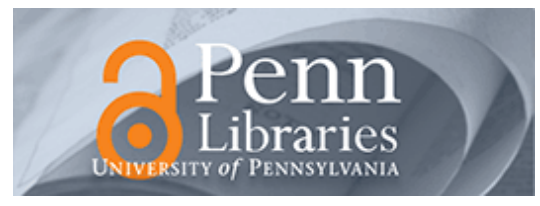

University of Pennsylvania ScholarlyCommons

$5-7-2012$

\title{
Experimental Verification of Displacement-Current Conduits in Metamaterials-Inspired Optical Circuitry
}

Brian Edwards

University of Pennsylvania

Nader Engheta

University of Pennsylvania, engheta@ee.upenn.edu

Follow this and additional works at: https://repository.upenn.edu/ese_papers

Part of the Electrical and Computer Engineering Commons

\section{Recommended Citation}

Brian Edwards and Nader Engheta, "Experimental Verification of Displacement-Current Conduits in Metamaterials-Inspired Optical Circuitry", . May 2012.

Edwards, B. \& Engheta, N. (2012). Experimental Verification of Displacement-Current Conduits in MetamaterialsInspired Optical Circuitry. Physical Review Letters, 108(19), 193902. doi: 10.1103/PhysRevLett.108.193902 (C)2012 American Physical Society

This paper is posted at ScholarlyCommons. https://repository.upenn.edu/ese_papers/610

For more information, please contact repository@pobox.upenn.edu. 


\title{
Experimental Verification of Displacement-Current Conduits in Metamaterials- Inspired Optical Circuitry
}

\author{
Abstract \\ Electric displacement current is present in capacitors and optical waveguides; however, unlike the \\ conduction current in metallic wires, it is not confined. Analogous to the contrast in conductivity between \\ a metallic wire and the surrounding air, displacement-current wires based on near-zero permittivity media \\ contain a large contrast in effective permittivity. As a variation on this idea, in this Letter, we demonstrate \\ at microwave frequencies two displacement-current cables based on effectively negative and effectively \\ positive permittivity metastructures. Our experimental results clearly show cablelike behaviors that allow \\ bending of the structure while still confining and maintaining the primarily longitudinal forward and \\ reverse effective displacement currents within each conduit. The results presented here experimentally \\ verify the notion of displacement-current wires and cables in metatronics as metamaterial-inspired \\ circuitry.

\section{Disciplines} \\ Electrical and Computer Engineering

\section{Comments} \\ Edwards, B. \& Engheta, N. (2012). Experimental Verification of Displacement-Current Conduits in \\ Metamaterials-Inspired Optical Circuitry. Physical Review Letters, 108(19), 193902. doi: 10.1103/ \\ PhysRevLett.108.193902 \\ (C2012 American Physical Society
}


Experimental Verification of Displacement-Current Conduits in Metamaterials-Inspired Optical Circuitry

\author{
Brian Edwards and Nader Engheta* \\ Department of Electrical and Systems Engineering, University of Pennsylvania, Philadelphia, Pennsylvania 19104, USA
}

(Received 2 November 2011; published 7 May 2012)

\begin{abstract}
Electric displacement current is present in capacitors and optical waveguides; however, unlike the conduction current in metallic wires, it is not confined. Analogous to the contrast in conductivity between a metallic wire and the surrounding air, displacement-current wires based on near-zero permittivity media contain a large contrast in effective permittivity. As a variation on this idea, in this Letter, we demonstrate at microwave frequencies two displacement-current cables based on effectively negative and effectively positive permittivity metastructures. Our experimental results clearly show cablelike behaviors that allow bending of the structure while still confining and maintaining the primarily longitudinal forward and reverse effective displacement currents within each conduit. The results presented here experimentally verify the notion of displacement-current wires and cables in metatronics as metamaterial-inspired circuitry.
\end{abstract}

DOI: 10.1103/PhysRevLett.108.193902

In radio-frequency (rf) electronics, simple metallic wires play an important role in connecting different lumped elements in a circuit, and thus they function as "conduits" for the flow of charged carriers and conduction currents [1-3]. Metals, due to their high conductivity, $\sigma$, at the rf frequencies, when surrounded by zero- (or low-) conductivity insulators, provide a medium with a high contrast in electric conductivity, leading to the confinement of conduction currents, $\sigma \boldsymbol{E}$ [3]. This seemingly simple and yet powerful combination of materials allows one to bend, twist, and stretch metallic wires in electronic circuits without significant leakage of current into the surrounding medium. In addition to the conduction current, one also encounters the electric displacement current, $\frac{\partial \boldsymbol{D}}{\partial t} \equiv$ $-i \omega \boldsymbol{D}=(-i \omega \varepsilon) \boldsymbol{E}$ (with $e^{-i \omega t}$ as the time convention), where $\boldsymbol{D}$ is the electric displacement vector, $\boldsymbol{E}$ is the electric field, and $\varepsilon$ is the local material permittivity $[4,5]$. In higher-frequency systems such as THz, IR, and visible wavelengths, displacement current plays a more dominant role than the conduction current. While materials with exceedingly low conductivities are readily available, the permittivity is lower-bounded by $\varepsilon_{0}$ in most conventional dielectrics. Therefore, a natural question arises: Can we devise a structure that can confine the displacement current, analogous with the way metallic wires confine conduction current? In our earlier theoretical work [6-9], we offered a solution to this problem using the concept of epsilon-near-zero (ENZ) metamaterials [10,11]. In such an "optical nanocircuit board" [9], the substrate was assumed to be made of an ENZ material out of which a set of airfilled (or, in general, dielectric-filled) grooves were carved. Since the local relative permittivity of the substrate was near zero, the displacement current was also zero in the ENZ substrate, leaving the nonzero displacement current confined within the grooves. Such grooves, therefore, act
PACS numbers: 42.82.Et, 52.40.Db, 52.40.Fd, 78.66.Sq

as displacement-current wires or, in short, "Ddot wires," where "dot" refers to the time derivative. This concept may offer exciting possibilities in design of optical circuitry at the nanoscale, providing a richer paradigm for the development of our metamaterial-inspired optical nanocircuitry ("metatronics") [6-8].

In the present Letter, we experimentally realize and verify a variation of the Ddot wire concept. Here, we realize the Ddot "coaxial" cable at the microwave frequencies, which is similar to the Ddot wire in its longitudinal displacement current, but it additionally contains both a forward and a reverse effective displacement current. By exploiting the dispersion characteristics of the hybrid mode in a parallel-plate waveguide with a dielectric slab, we create regions with tunable effective material parameters. By rendering regions outside the slab (but within the parallel-plate waveguide) to be acting effectively as plasmonic regions, we confine the prominently longitudinal Ddot current to within the slab, while the return Ddot current flows back in the region around this slab. Our experimental results, which are in full agreement with our full-wave simulations, clearly show that the Ddot currents may indeed be confined within two regions with contrast in their effective relative permittivities, and such confinement is robust in both straight and serpentine geometries. (A video summary of our present work can be found in the Supplemental Material [12].)

Figure 1 shows the geometry of one of our experimental setups. This waveguiding structure is formed by two parallel brass plates, assumed to be perfect electrical conductors, separated with a height of $a=50.8 \mathrm{~mm}$, with open boundaries at its two sides. A dielectric fin made of crosslinked polyethylene (Eccostock ${ }^{\mathrm{TM}} \mathrm{CPE}$ ) with permittivity $\varepsilon_{\text {diel }}=2.4\left(1+i 7 \times 10^{-4}\right) \varepsilon_{o} \quad[13]$, height of $50.8 \mathrm{~mm}$, and a width of $b=63.3 \mathrm{~mm}$ is inserted 
in this waveguide. A cross section of this setup, shown as the inset in Fig. 1, consists of the central dielectric region with $\varepsilon_{\text {diel }}$ and the two outer air regions with $\varepsilon_{o}$, all contained between the two parallel plates which are assumed to extend infinitely for the sake of simplicity in the analytical modeling. While there are several possible guided modes for such a geometry, we work with only the hybrid mode with longitudinal components

$$
E_{x}(x, y, z)= \begin{cases}E_{1} e^{i k_{x} x-\gamma_{z} z} \sin (\pi y / a) & z>b / 2 \\ E_{2} e^{i k_{x} x} \cos \left(z k_{z}\right) \sin (\pi y / a) & -b / 2<z<b / 2 \\ E_{1} e^{i k_{x} x+\gamma_{z} z} \sin (\pi y / a) & z<-b / 2\end{cases}
$$

and

$$
H_{x}(x, y, z)= \begin{cases}H_{1} e^{i k_{x} x-\gamma_{z} z} \cos (\pi y / a) & z>b / 2 \\ H_{2} e^{i k_{x} x} \sin \left(z k_{z}\right) \cos (\pi y / a) & -b / 2<z<b / 2 \\ -H_{1} e^{i k_{x} x+\gamma_{z} z} \cos (\pi y / a) & z<-b / 2,\end{cases}
$$

where $E_{1}, E_{2}, H_{1}$, and $H_{2}$ are complex coefficients describing the amplitude and phase of the fields and $k_{x}, k_{y}, k_{z}$, and $\gamma_{z}$ are propagation constants. From these components, all other field components can be calculated through Maxwell's equations [14]. If we confine our observation to the middle plane, i.e., the $x$-z plane where $y=a / 2$, an effective relative dielectric constant can be assigned to field propagation in the three regions by examining the propagation constants in each region and drawing an analogy to that of an effective plane wave propagation along this $x-y$ plane. This is a well-known technique in microwave design [15], which leads to the following results for the effective relative permittivity values:

$$
\varepsilon_{\text {rel,eff }}= \begin{cases}\left(k_{x}^{2}-\gamma_{z}^{2}\right) / k_{0}^{2}=1-\pi^{2} /\left(a^{2} k_{0}{ }^{2}\right) & z>b / 2 \\ \left(k_{x}^{2}+k_{z}^{2}\right) / k_{0}^{2}=\varepsilon_{\text {diel }}-\pi^{2} /\left(a^{2} k_{0}{ }^{2}\right) & -b / 2<z<b / 2 \\ \left(k_{x}^{2}-\gamma_{z}{ }^{2}\right) / k_{0}^{2}=1-\pi^{2} /\left(a^{2} k_{0}{ }^{2}\right) & z<-b / 2,\end{cases}
$$

where $k_{0} \equiv \omega \sqrt{\mu_{0} \varepsilon_{0}}$ is the free-space propagation constant.

For a given frequency and plate separation $a$, it is apparent that one can make the effective relative permittivity of a region positive or negative, depending on the choice of the filling materials. For instance, in our geometry, at the design frequency $f_{d}$, the outer regions are

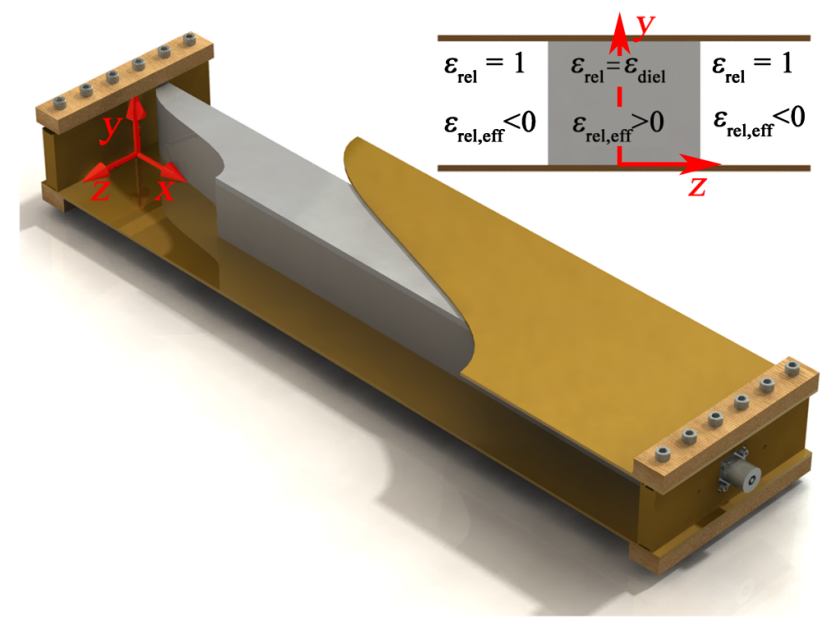

FIG. 1 (color online). Experimental setup consisting of a dielectric fin sandwiched between two metallic sheets. The region within the fin has an effectively positive permittivity, while that air region outside of the fin under the two copper plates has an effective negative permittivity. The desired mode is excited using two monopoles fed by 50-Ohm coaxial cables. designed to have a negative effective relative permittivity while the inner region is designed to have a positive effective permittivity. Therefore, we are able to exploit the dispersion characteristics of hybrid modes in such a waveguide to achieve wave characteristics with effective positive or negative relative permittivity, leading to interesting techniques in the design of structural metamaterials. In our earlier works, we have used similar techniques to produce structures with effective permittivity near zero, experimentally demonstrating some of the ENZ properties in the microwave domains [11].

The hybrid mode of interest here has a cutoff frequency below which propagation along the $x$ direction becomes evanescent [i.e., $\operatorname{Re}\left(k_{x}\right)=0$ ] and above which the mode is propagating $\left[\operatorname{Im}\left(k_{x}\right)=0\right]$. Assuming lossless materials, at the cutoff frequency, the propagation constant $k_{x}$ is ideally zero in both parts, meaning the guided wavelength along the $x$ direction is effectively infinite. We consider this cutoff frequency as our design frequency, $f_{d}$, which can be tailored via the proper choice of $a$ and $b$ for a given dielectric fin.

In our experimental setup, since the field is evanescent in the outer air regions between the two copper plates, one may truncate the geometry in the positive and negative $z$ directions without any substantial deviation from the ideal analytical results. Along the $x$ direction, the fields are excited and received via small monopole probes protruding from electrically conductive walls at each end. The length of these monopoles was optimized to approximately match 
the waveguide to the attached $50 \Omega$ cables, although no extensive efforts to match were made beyond that, since this is not a crucial point in our experiment. The waveguide dispersion relation yielded a design frequency of $f_{d}=$ $2.125 \mathrm{GHz}$ and, according to Eq. (3), an effective relative permittivity of $\varepsilon_{\text {rel,eff }}=0.47$ inside the dielectric region and $\varepsilon_{\text {rel,eff }}=-0.93$ outside the dielectric region. (We emphasize again that these are the two effective relative permittivities of the two regions, not the true permittivity values, which are $\varepsilon_{\text {diel }}$ and $\varepsilon_{o}$ for inside and outside the dielectric, respectively.)

Given that the dielectric is not ideally lossless, the propagation constant $k_{x}$ will never be identically zero. Using analytical results with realistic dielectric losses, in Fig. 2(c), we see that, at the design frequency, the propagation constant is $k_{x} / k_{0}=0.0268(1+i)$, meaning that, after 37 free-space wavelengths, the field will have undergone $2 \pi$ radians of phase change and be at $e^{-2 \pi}$ of its original amplitude. One should note that, while the relative bandwidth is quite small, arguably at optical frequencies within the field of metatronics, the absolute bandwidth may still be reasonably large. Moreover, the group velocity may also be small but not zero within the bandwidth of interest [16]. See [12] for further details.

We then simulated the electromagnetic field distributions in the full experimental structure using the commercial electromagnetic software CST Microwave Studio $^{\mathrm{TM}}[12,17]$, and the results are shown in the top panel of Fig. 2, where the vector plot is the real part of the electric field distribution at a moment in time. This confirms that the displacement current $-i \omega \varepsilon_{\text {eff }} \boldsymbol{E}$, not the conduction current $\sigma \boldsymbol{E}$ (since $\sigma \approx 0$ ), while predominantly longitudinal, "flows" along the structure, similar to what occurs in a capacitor. However, unlike a capacitor, this structure is almost four free-space wavelengths long at the design frequency. Because of imperfect matching, there are both forward and weaker backward traveling wave components superimposed on top of one another, both of the form given in Eq. (1). Despite this minor complication due to imperfect matching, it is apparent from the simulation that the phase changes relatively little from one end of the structure to the other. As shown in panels (a) and (b), plotting the magnitude and phase of the electric field along the line $S$ supports this, showing a variation of only about $\pi$ radians across nearly four free-space wavelengths. So, this indeed acts as a "capacitor," whose plates are separated four wavelengths long, and the electric field still stays longitudinal along the capacitor without leaking into the air region.

Experimental measurements of the longitudinal component of the electric field $E_{s}$ were taken along the indicated line $\mathbf{S}$ near the dielectric fin using a small dipole antenna. The amplitude and phase of $E_{s}$ measured at $0.993 f_{d}$ were compared with the simulation results at $f_{d}$ in the left and middle insets of Fig. 2. The agreement with numerical simulation is quite good. The phase varies by a value under

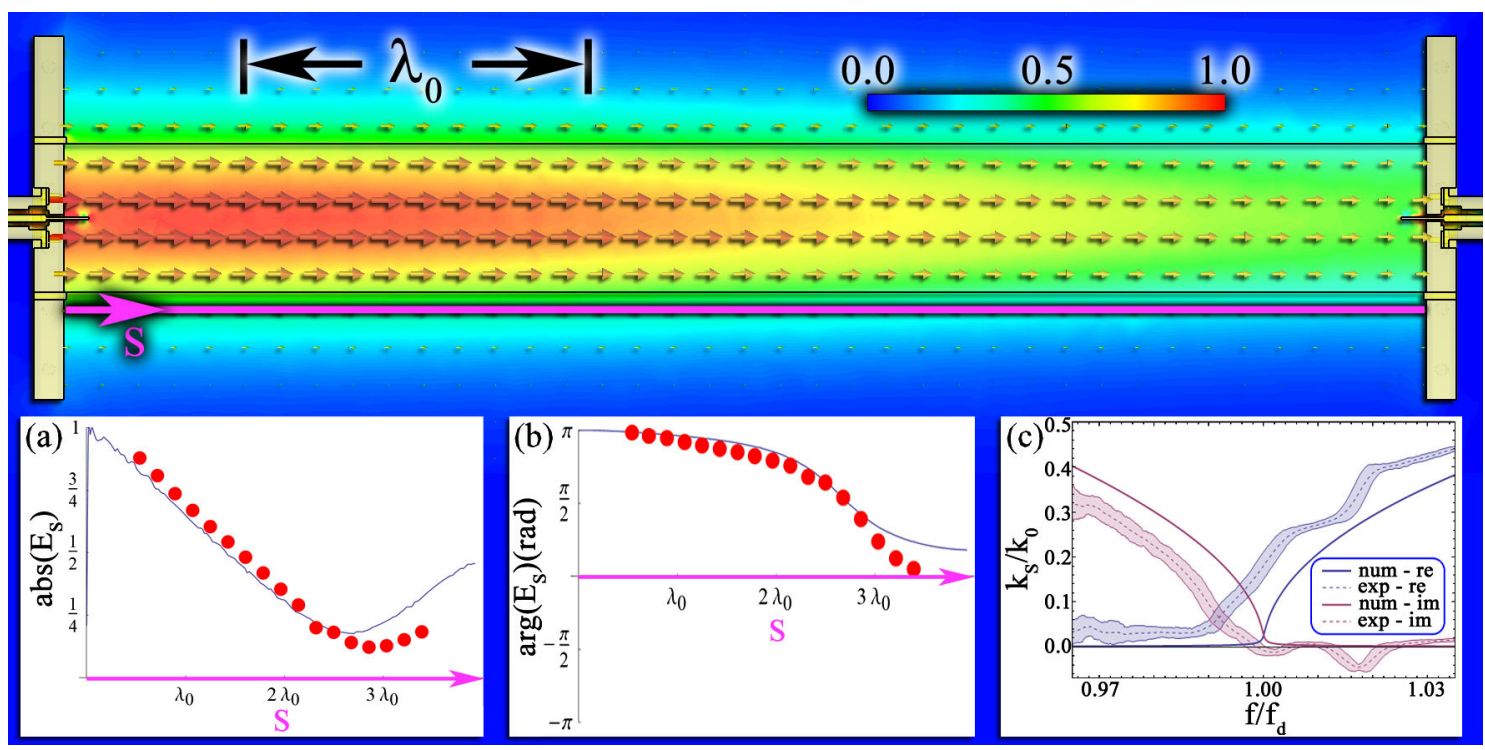

FIG. 2 (color online). Comparison between the simulated and experimental electric field distributions measured along the structure. Top panel: The real part of the electric field from our simulation is shown at the midplane of the structure. The electric field shows little phase variation, despite traveling almost $4 \lambda_{0}$. Additionally, it is longitudinal to the direction of conduit. Several measurements were taken along the line marked $S$ (red dots) and compared to simulation results (solid line) in (a) amplitude and (b) phase. These were used to find the experimental propagation constant $k_{S}$, shown in (c). In (c), the numerical results and the experimentally extracted values of the real and imaging parts of the propagation constant are shown. At frequencies below the design frequency, the field is largely evanescent, characterized by a $k_{S}$ which is largely imaginary. At frequencies above the design frequency, it is propagating, characterized by a $k_{S}$ which is largely real. However, at the design frequency, the field has little variation along $k_{S}$, as both the real and imaginary parts of the propagation constant are small. 
$\pi$ radians over approximately four free-space wavelengths in both simulation and experiment. By assuming that the measured field consists of a forward and a backward wave with variation $e^{i k_{\mathrm{S}} S}$, using the amplitude and phase of the measured results, one can calculate the propagation constant $k_{\mathbf{S}}$ along the line $\mathbf{S}$. This is shown in the right inset against the analytic results for $k_{x}$. In both theory and experiment, it is clear that there is a point in which minimal attenuation [i.e., small $\left.\operatorname{Im}\left(k_{x}\right)\right]$ and minimal phase change [i.e., small $\left.\operatorname{Re}\left(k_{x}\right)\right]$ may be achieved. The small frequency shift between these two minima justifies the use of $0.993 f_{d}$ in the left and middle insets and can be attributed to small experimental imperfections in the fabrication.

The longitudinal electric field is strongest at the center of the cross section. This is shown in the analytical result in Fig. 3(a). To verify this, measurements were taken at the locations indicated. Since half of these measurements were inside the dielectric itself, a small slot was cut, into which the dipole probe was inserted. Simulations were used to find a correction factor for the probe dipole gain when inside the dielectric slot. It is clear from Fig. 3(b) that the measured fields follow the analytical prediction nicely.

The effective electric displacement current $D$ can be found by multiplying both the measured electric fields and the analytical electric fields by the effective permittivity $\varepsilon_{\text {eff }}$ calculated in Eq. (3). It can be seen that inside the dielectric region there is a strong "forward" displacement current and outside the dielectric there is a "reverse" displacement current giving the structure a "coaxial" quality. Just as a coaxial waveguide has equal forward and backward current on any cross section, we find the same using the effective displacement current. Integrating the effective electric displacement current across the structure ( $\left.I=\int_{-\infty}^{\infty}-i \omega \varepsilon_{\text {eff }} E d z\right)$, we find that it is indeed zero, confirming that indeed the structure behaves as a "coaxial cable" for the displacement current. This is not surprising, since the magnetic field is zero at large distances in the $+z$ and $-z$ directions. Nevertheless, it speaks to the utility and validity of the concept of the effective permittivity that this yields the expected results.

A waveguide ceases to transmit energy when either the guided mode is converted into other propagating modes or it is lost as heat. These other propagating modes may carry energy away from the guide in the form of radiation such as occurs in a fiber optic cable which is bent too harshly or they may reflect energy into the backward reflected wave as occurs in a kinked rf cable. Generally, a wave will survive an abruption if the guided wavelength is much longer than the abruption. For this reason, the common household power cord does an amazing job of guiding current and energy, despite tremendous abuses along the way. Our structure is uniquely positioned to bring some of these useful properties of the wire into dimensions which are typically associated with waveguides. By making the guided wavelength substantially longer than the free-space
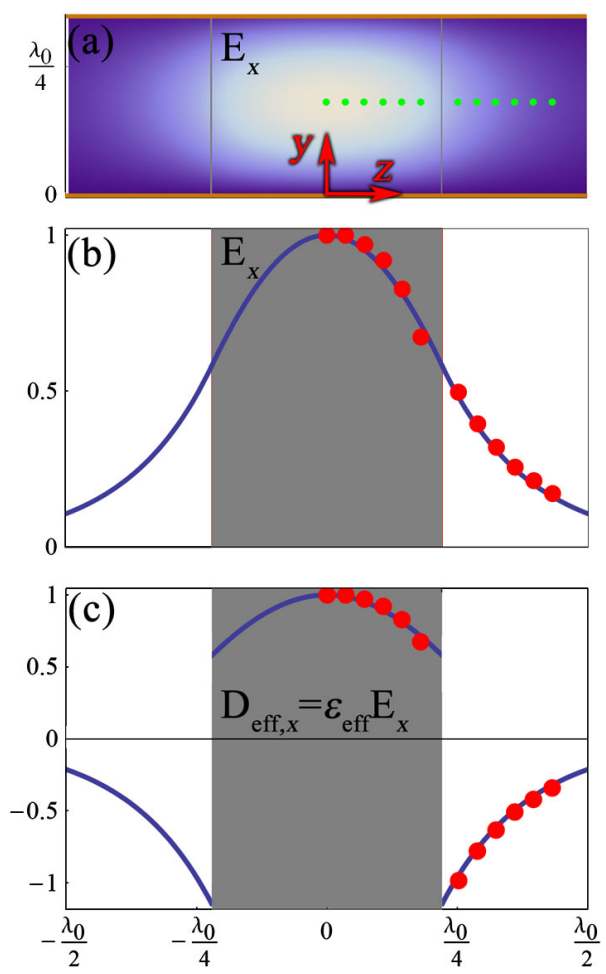

FIG. 3 (color online). Comparison between the analytical and experimental electric field distributions across the dielectric fin as the Ddot cable. (a) The amplitude of the longitudinal component of the electric field, $E_{x}$, was found analytically and shown on the $y-z$ plane [solid lines in (b)]. It is strongest at the center of the fin, varies sinusoidally in the fin, and then decays evanescently in the air region between the parallel plates (this air region has an effectively negative permittivity, while its actual permittivity is that of air) on either side. Measurements were taken at the positions indicated by the green dots in (a), and the measured results are shown as the red dots in (b). When both the analytical and measured fields are multiplied by the analytically obtained effective permittivity from Eq. (3), the "coaxial cable" nature of the Ddot cable becomes apparent, showing a forward effective displacement current within the fin and an equal but backward effective displacement current in the effectively negative permittivity regions to either side.

wavelength $\lambda_{0}$, all abruptions may appear small. The ability of the Ddot cable's guided mode to survive bends and abruptions has been experimentally verified in another set of experiments described below.

While the geometry in Fig. 1 provides concise analytical results, a serpentine structure as seen in Fig. 4 demonstrates the structure's "guiding" abilities as a "conduit" for the displacement current more readily. In this experimental setup, we have built the dielectric fin to have a "wavy" shape, as seen in Fig. 4. See [12] for details. The width and the height of this serpentine fin are the same as those of the straight fin in the first setup. However, its length has been increased to eight free-space wavelengths. As we can see in the simulations in Fig. 4, we have under $\pi / 2$ radians of phase variation along such a long length of 


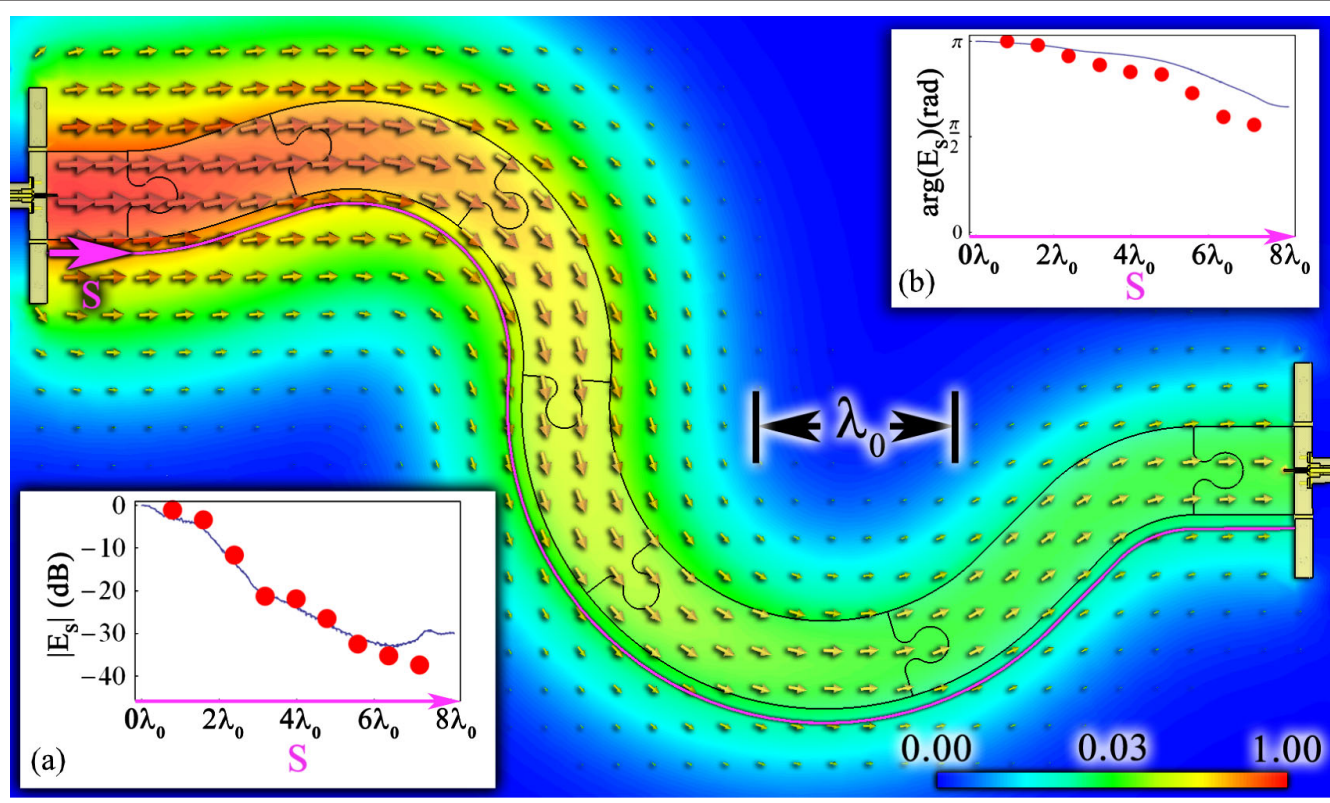

FIG. 4 (color online). Simulated and experimental electric field distributions on a serpentine fin as the wavy Ddot cable. The simulated $\operatorname{Re}(E)$ at the midplane is shown, clearly demonstrating the longitudinal nature of the field. The experimental (red dots) and numerical (solid line) results for the magnitude and phase of the electric fields are shown in the insets. The measurement was made at nine locations along the curve marked $S$. Despite the fact that, near the design frequency, the length of this Ddot cable is approximately eight free-space wavelengths, the phase of the field varies little.

wavy fin. Note again that the vector plot denotes $\operatorname{Re}(E)$ and not the Poynting vector. The electric field follows the fin with small linear phase variation along its length, despite several curves, demonstrating the fact that indeed the structure behaves as a Ddot cable.

A dipole probe was used to measure the electric field along the line $S$. The amplitude and the phase of these measured fields are shown in the insets of Fig. 4 and compared to numerical simulation with good agreement. It is apparent from the analytical results in Fig. 2(c) that, for finite losses, one has the choice of lower phase variation [i.e., lower $\operatorname{Re}\left(k_{S}\right)$ ] at the expense of greater attenuation [i.e., greater $\left.\operatorname{Im}\left(k_{S}\right)\right]$. In this case, we highlight a frequency slightly below $f_{d}$ in order to gain lower phase variation along the structure.

In conclusion, in two sets of experiments in the microwave regime, we have demonstrated that the displacement current can indeed be tailored and shaped using properly designed structures, analogous to what metallic wires can do for the conduction current. Since the concept of Ddot conduits is an important part of the field of metatronics, in which the nanoparticles can be "connected" via the Ddot wires and cables to form metamaterial-inspired optical nanocircuitry, this present Letter reveals the proof of this concept in the microwave domain. This will open up exciting possibilities for the design of devices and components that can benefit from such Ddot conduits.

This work is supported in part by the U.S. Air Force Office of Scientific Research (AFOSR) Grants No. FA9550-08-1-0220 and No. FA9550-10-1-0408.
*To whom correspondence should be addressed. engheta@ee.upenn.edu

[1] J. D. Irwin, Basic Engineering Circuit Analysis (PrenticeHall, New York, 1996), 5th ed.

[2] D. M. Pozar, Microwave and RF Design of Wireless Systems (Wiley, New York, 2001).

[3] Ben G. Streetman and Sanjay Banerjee, Solid State Electronic Devices (Prentice-Hall, New Jersey, 2000), 5 th ed.

[4] J.C. Maxwell, A Treatise on Electricity and Magnetism (Dover, New York, 1954), Vols. 1 and 2.

[5] J. D. Jackson, Classical Electrodynamics (Wiley, New York, 1999), 3rd ed.

[6] N. Engheta, Science 317, 1698 (2007).

[7] N. Engheta, Phys. World 23, 31 (2010).

[8] N. Engheta, A. Salandrino, and A. Alu, Phys. Rev. Lett. 95, 095504 (2005).

[9] A. Alu and N. Engheta, Phys. Rev. Lett. 103, 143902 (2009).

[10] M. G. Silveirinha and N. Engheta, Phys. Rev. Lett. 97, 157403 (2006).

[11] B. Edwards, A. Alu, M.E. Young, M. Silveirinha, and N. Engheta, Phys. Rev. Lett. 100, 033903 (2008).

[12] See Supplemental Material at http://link.aps.org/ supplemental/10.1103/PhysRevLett.108.193902 for a detailed description of the materials and methods of both experiment and numerical simulations.

[13] Eccostock CPE, Emerson \& Cuming Microwave Products, www.eccosorb.com.

[14] C. A. Balanis, Advanced Engineering Electromagnetics (Wiley, New York, 1989), 3rd ed.

[15] W. Rotman, IRE Trans. Antennas Propag. 10, 82 (1962).

[16] M. Silveirinha and N. Engheta, Phys. Rev. B 76, 245109 (2007).

[17] CST Studio Suite ${ }^{\mathrm{TM}}, 2010$, www.cst.com. 